\title{
Auditory Cortex
}

National Cancer Institute

\section{Source}

National Cancer Institute. Auditory Cortex. NCI Thesaurus. Code C52712.

The area in the cerebral cortex that receives and processes auditory input. 